\title{
Surround Sound Spreads Visual Attention and Increases Cognitive Effort in Immersive Media Reproductions
}

\author{
Catarina Mendonça* \\ Department of Psychology, University of Azores \\ Center for Psychology, University of Porto \\ Ponta Delgada, Portugal \\ ana.cm.hiipakka@uac.pt
}

\author{
Victoria Korshunova \\ HumanTechnology Interaction, Eidhoven University of \\ Technology \\ Eidhoven, Netherlands \\ v.korshunova@tue.nl
}

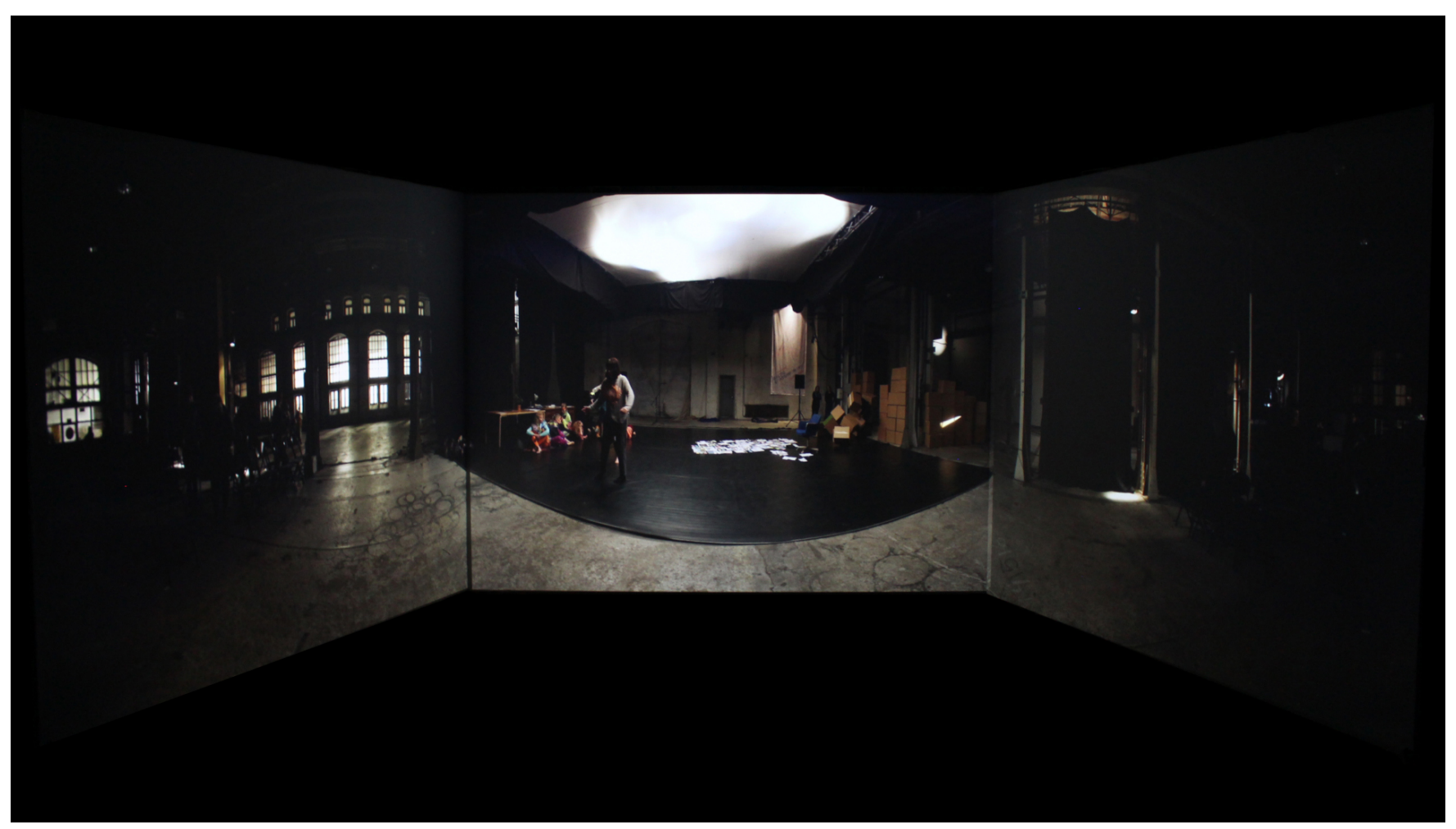

Figure (1) Screenshot of the immersive video being reproduced.

\begin{abstract}
The goal of this study was to explore the effects of different spatial sound configurations on visual attention and cognitive effort in an immersive environment. For that purpose, different groups of people were exposed to the same immersive video, but with different soundtrack conditions: mono, stereo, 5.1 and 7.4.1. The different sound conditions consisted of different artistic adaptations of the same soundtrack. During the visualization of the video, participants

\footnotetext{
${ }^{*} \mathrm{CM}$ planned the project, analysed data and wrote the paper. VK programmed the experiments, ran the experiments, and analysed data.
}

\section{(c) (†)}

This work is licensed under a Creative Commons Attribution International 4.0 License. AM'20, September 15-17, 2020, Graz, Austria

(C) 2020 Copyright held by the owner/author(s).

ACM ISBN 978-1-4503-7563-4/20/09.

https://doi.org/10.1145/3411109.3411118 wore an eye-tracking device and were asked to perform a counting task. Gaze direction and pupil dilation metrics were obtained, as measures of attention and cognitive effort. Results demonstrate that the conditions 5.1 and 7.4.1 were associated with larger distributions of the visual attention, with subjects spending more time gazing at task-irrelevant areas on the screen. The sound condition which led to more concentrated attention on the task-relevant area was mono. The wider the spatial sound configuration, the greater the gaze distribution. Conditions 7.4.1 and 5.1 were also associated with larger pupil dilations than the mono and stereo conditions, showing that these conditions might lead to increased cognitive demand and therefore increased task difficulty. We conclude that sound design should be carefully planned to prevent visual distraction. More surrounding spatialized sounds may lead to more distraction and more difficulty in following audiovisual contents than less distributed sounds. We propose that sound spatialization and soundtrack design should be adapted to the audiovisual content and the task at hand, varying in immersiveness accordingly. 


\section{CCS CONCEPTS}

- Human-centered computing $\rightarrow$ Mixed / augmented reality; Sound-based input / output; Auditory feedback; Activity centered design;

\section{KEYWORDS}

virtual reality, sound design, audiovisual, spatial audio, perception, attention, difficulty, pupil dilation

\section{ACM Reference Format:}

Catarina Mendonça and Victoria Korshunova. 2020. Surround Sound Spreads Visual Attention and Increases Cognitive Effort in Immersive Media Reproductions. In Proceedings of the 15th International Audio Mostly Conference (AM'20), September 15-17, 2020, Graz, Austria. ACM, New York, NY, USA, 6 pages. https://doi.org/10.1145/3411109.3411118

\section{INTRODUCTION}

Over the past decades we have witnessed an improvement in the realism and quality of immersion in multimedia reproductions. Sound reproduction systems have become more surrounding, with more spatially defined virtual auditory objects, which are capable of conveying very vivid and realistic impressions. Combined with ever expanding high resolution video displays, audiovisual reproductions are now more immersive than ever and reaching the average consumer. It is broadly accepted, in audiovisual reproductions, that the more immersive the technology is, the better the user experience [3]. However, there are many aspects to user experience which should be carefully addressed and considered. In the study reported here we approach specifically the issue of attention in immersive audiovisual systems.

Sounds can involuntarily guide visual attention [11], which might enhance the detection of, and response to, visual stimuli within the attended area. Preliminary studies with immersive video have shown that sound can cause some visual attention effects. Auditory cueing, in the form of informative soundtracks starting before the visual scene, guides visual attention and helps in the detection of visual events [14]. These effects are particularly relevant for events in the visual periphery and with informative soundtracks. In another study, where subjects are asked to focus only in one specific event of the audiovisual reproduction - it was found that participants made more errors counting visual events when the soundtrack was fully immersive and surrounding, as opposed to in less spatialized mono and stereo conditions [13]. That same study showed that participants observed larger areas of the screen and were therefore less able to focus specifically on the relevant screen area when more enveloping 3D sound was used. A more recent study replicated those findings using different soundtrack immersiveness levels in a movie reproduction [9]. It was found that subjects spent more time looking at non task-related screen areas with surrounding sound conditions, as opposed to when the sound came exclusively from the central part of the screen.

Here, we took this question further, and were interested in analysing if focusing on visual events in a movie scene became easier or harder under different sound spatialization conditions. The soundtrack was informative and spatially congruent with the video, and four audio reproduction setups were tested: mono (1 speaker), stereo (2 speakers), 5.1 ( 5 speakers and a subwoofer) and 7.1.4 (11 speakers and a subwoofer). Nowadays, surround sound condition 5.1 and spatial audio condition 7.1.4 are becoming increasingly popular and are more likely to be found in regular households. To test task difficulty, participants wore eye-tracking glasses during the visualization of the immersive video. Gaze direction and pupil dilation were measured throughout time. Pupil dilation provides a direct physiological measure of mental or cognitive effort, where the more dilation the greater the effort in the task at hand [15]. Following the findings from Mendonça and colleagues [13] it was hypothesized that in more immersive and surrounding sound conditions there would be greater cognitive effort.

\section{METHODS}

\subsection{Participants}

There were 54 participants ( 12 females, 42 males) in this experiment. All participations were voluntary and no compensation was offered. The data of two participants ( 1 female, 1 male) were discarded due to technical failures at the beginning of the experiment (no data recorded). The data of 52 participants were thus considered in the statistical analysis. The participants had ages ranging between 20 50 years (31.68+/- 6.84 years). All participants had normal vision or corrected-to- normal vision. Those with corrected-to-normal vision were in a range from around +3 farsighted and around 7.5 nearsighted. All participants reported having normal hearing and were naive regarding the purpose of the study. Besides the 54 participants who participated in the experiment, three additional participants, who were familiar with the experiment and the stimuli, took a part in an informal pilot study to secure the functioning of the experimental setup. These informal data were not included in the statistical analysis of the experiment. All 54 participants gave informed consent as to their participation after the purpose and procedure of the experiment was explained to them. The experimental procedure and the collection of personal data were guided and approved by the Aalto University Ethics Committee.

\subsection{Settings}

The tests were performed in an immersive audiovisual environment at the Department of Signal Processing and Acoustics at the Aalto University School of Electrical Engineering. The setting was situated inside an acoustically treated space and comprised three HD video projectors and 40 loudspeakers (Genelec 1029). The screens were $2.5 \mathrm{~m}$ by $1.88 \mathrm{~m}$ each and they were disposed in a pentagonal shape, where two sides of the pentagon were absent (see Figure 2). The resolution of the video material was transformed into 4320 by 1080 pixels. More specific details can be found in [1]. The audio system arrangement for mono, stereo, 5.1 and 7.1.4 was built based on the information of azimuths, elevation and distance from the audio control booth used for mixing and mastering. It was equipped with Avid Pro Tools 12 HDX2 system and Mac OS, 16 channels Avid S6 control surface, a $2 \mathrm{~m}$ by $2.7 \mathrm{~m}$ projection screen and a 9.1.4 Dolby Atmos speaker setup with Genelec speakers. During mixing, plugin Spanner was one of the main panning instruments for complete independent control of every channel. We used 12 loudspeakers out of 40 available for this test. Participants interacted with the system via a tablet computer with a touch screen interface. This interface was developed specifically for the experiment with a Max/MSP visual programming language. 

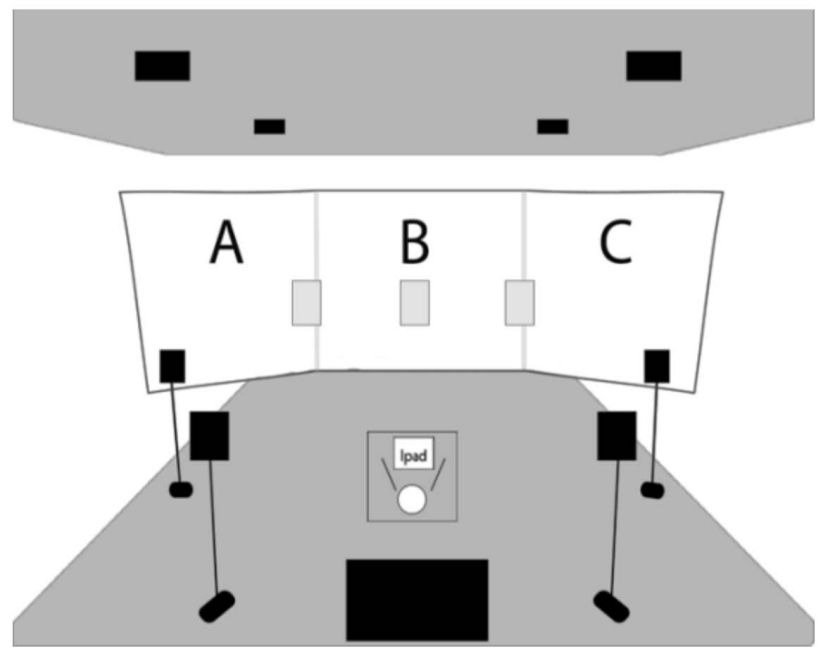

Figure (2) Representation of the settings where the audiovisual experiment took place. There were three display areas $(A, B, C)$ and four sound system configurations (mono, stereo, 5.1 and 7.4.1)

\subsection{Stimuli}

The visual stimulus consisted of a 9-second long scene from a 3D movie provided by author Synes Elischka, a Doctoral Candidate at the Department of Film, Television and Scenography in Aalto University. The stimulus can be downloaded here [12]. The movie is called Ego Cure [6] and it is the first VR film made in Finland. It was shot on $360 \mathrm{RED}$ Epic cameras with a $6 \mathrm{~K}$ high-quality footage. The sound was recorded with boom microphones and standard audio recording techniques for movie production. The movie was a pilot project produced in 2017 by Aalto University ELO Film School Helsinki in collaboration with Aalto University Medima Lab. Originally it was created for a VR headset and binaural headphone listening mixed by sound designer Jorma Kaulanen. In total, over sixty people including professional freelancers and students were involved in the production of Ego Cure. In the visual stimulus, participants could see a large space with people sitting or moving on the left side of the middle display. There were falling boxes on the right side of the middle display. This stimulus was accompanied by four different audio conditions: mono, stereo, 5.1 and 7.1.4. All conditions were professionally mixed and mastered in a sound studio. The idea was to create less point-like and more diffused audio outside the task-related area, i.e., the display (B) that participants needed to view in order to perform the task. Parameters of the studio (azimuths, elevation and distance) were provided and closely replicated in the experimental audiovisual environment. The mono condition reproduced the soundtrack using only one loudspeaker, behind the main screen in the center. The stereo condition reproduced the soundtrack with two loudspeakers, one between screen A and B and the other between screen B and C (see Figure 2). The 5.1 and 7.4.1 conditions followed the standard loudspeaker distributions for these configurations [2]. Condition 5.1 used 5 loudspeakers at the head level, one behind the screen, and a subwoofer. Condition 7.4.1 used 7 loudspeakers at head level, 4 above the head, and a subwoofer.

\subsection{Test Procedures}

In this study, four groups of 13 participants performed a selective visual attention task, while watching a scene from the VR movie Ego Cure [6], with one out of the four audio conditions mentioned above. The participants were asked to count how many falling boxes they could see in the scene. This task was explained before the playback of the movie. During the task, the participant's gaze direction and pupil dilation were tracked by an eye-tracking system (Tobii Pro 2 Glasses).

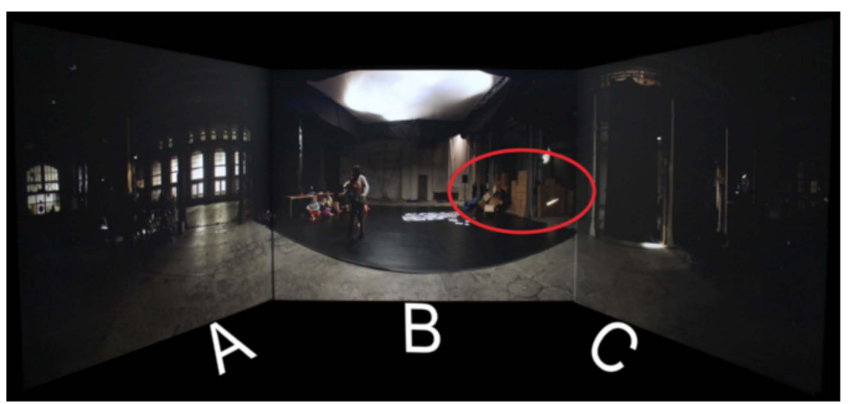

Figure (3) The area of interest. The area in the screen participants had to look at in order to perform the counting task is marked with a red elipse.

Each participant was seated at the center of the audiovisual system and had a tablet computer with a touch screen (Apple iPad Mini) in their hands. During the whole experiment, participants wore the Tobii Pro 2 eye-tracking glasses which allows participants to move their head freely while watching the displays. This eye-tracking device also recorded sound, video, and head coordinates for data analysis. The participants were asked to perform a visual selective attention task: counting how many falling boxes they could see in the movie scene (Figure 3 for a snapshot). The total number of falling boxes was 17 . The fifty-two participants were randomly divided into the four groups. Before the start of the experiment, each participant practiced a trial with no audio and completely different visual stimuli. Still pictures with white circles on a black background were used as examples to familiarize participants with the Max/MSP and tablet interface so as to prevent questions and mistakes. None of the participants saw the original material used for the main experiment. The playback of used audiovisual stimulus was under control of the participant. When the participant was ready, he/she could touch a Play video button and read the question: "How many falling boxes can you see? Watch the video and input the total number of counted boxes". The average duration of the experiment was 15 minutes per participant.

\section{RESULTS}

\subsection{Gaze Analysis}

For the purpose of gaze analysis, all eye-tracking data were classified into fixations and pursuits. Fixations are defined as maintaining the eye on a single location, while pursuits relate to when the eye is moving. It is only during a fixation that the subjects actually attentively see a target and acquire meaningful information about it. Figure 4 shows the distribution of fixations during the visualization of the immersive video scene, in each of the sound conditions. 
Table (1) Size of area of fixation $\left(\mathrm{m}^{2}\right)$ per sound condition

\begin{tabular}{lccccc}
\hline Nr. fixations & $>100$ & $>200$ & $>300$ & $>400$ & $>500$ \\
\hline Mono & 4.93 & 0.19 & 0.04 & 0.02 & 0.002 \\
Stereo & 5.05 & 0.16 & 0.19 & 0.07 & 0.025 \\
5.1 & 5.23 & 0.27 & 0.22 & 0.09 & 0.028 \\
7.4 .1 & 5.49 & 0.34 & 0.22 & 0.15 & 0.028 \\
\hline
\end{tabular}

The frequency of the fixations is color coded in a heat map where colors represent the number of total fixations across participants per condition. Transparent green corresponds to 100-200 fixations, solid green corresponds to 200-300 fixations, yellow corresponds to 300-400 fixations, orange corresponds to 400-500 fixations, and red corresponds to more than 500 fixations.

It is noticeable that the non task-related screens (the left and right screens) were more explored in the 5.1 condition and even more so in the 7.4.1 condition.The Mono condition had the most gaze fixations concentrated in the area of interest. To better quantify the areas where participants distributed their fixations in each sound condition, a table is presented with the total area where a minimum number of fixations occurred in each sound condition (Table 1).

It is observed that the area where at least 100 fixations occurred is very large overall, and the more enveloping the sound condition the wider the area attended. This trend was observed also for the remaining concentrations of fixations analyzed. It can therefore be concluded that more immersive sound conditions lead to subjects paying attention to a wider area on the screen, even when they are asked to concentrate on a specific point. More fixations are found in task-irrelevant areas when sound is more immersive. Conversely, mono sound leads to lower exploration of the visual screen and greater focus on the area of interest.

\subsection{Pupil Dilation}

In this section, we take a look at the average diameter of pupils throughout time in each sound condition. For this analysis, both eyes were averaged per subject and missing frames filled with an interpolation function. Figure 5 shows how the average pupil diameter changed throughout time since the first second of the scene, which was taken as baseline. The graph shows only the data from the moment boxes started falling onward. There was no change in screen luminance throughout the scene. As can be observed, pupils dilated in the first three seconds of boxes falling, then showing a trend toward stabilization. After one second of the beginning of the task, some differentiation between conditions started to occur. Five seconds into the scene, when the diameter of pupils begun to stabilize, it is observed that the lowest dilations occurred in the stereo condition, followed by the mono condition. Conditions 5.1 and 7.4.1 alternated in which led to more pupil dilation, overlapping curves at the end of the scene. More pupil dilation is considered to reveal more cognitive effort, so we can say that it was more difficult for the subjects to count the boxes in the 5.1 and 7.1.4 conditions.

A one-way analysis of co-variance (ANCOVA) was conducted to determine statistically significant differences between the four sound conditions on pupil dilations, controlling for time. There was a significant effect of sound condition on pupil dilation after

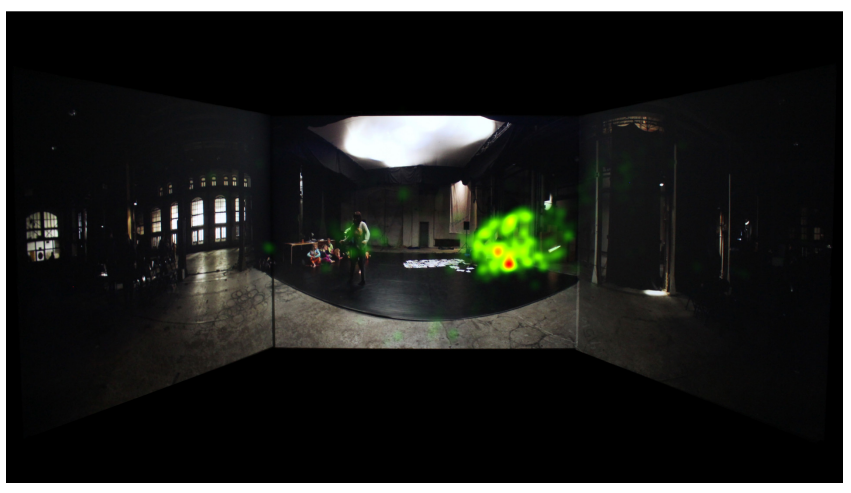

(a) Mono sound condition

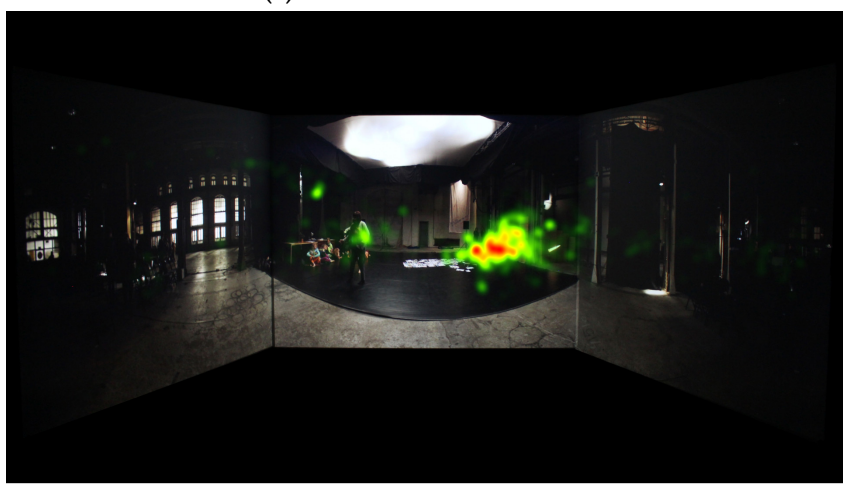

(b) Stereo sound condition

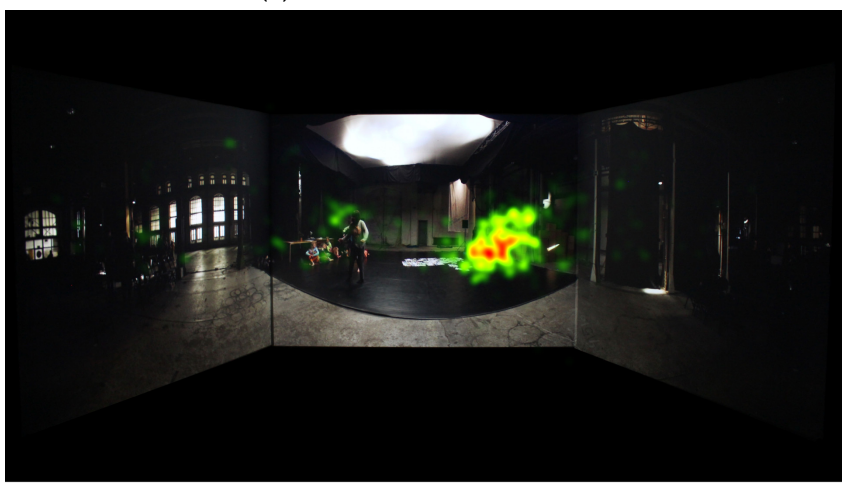

(c) 5.1 Sound Condition

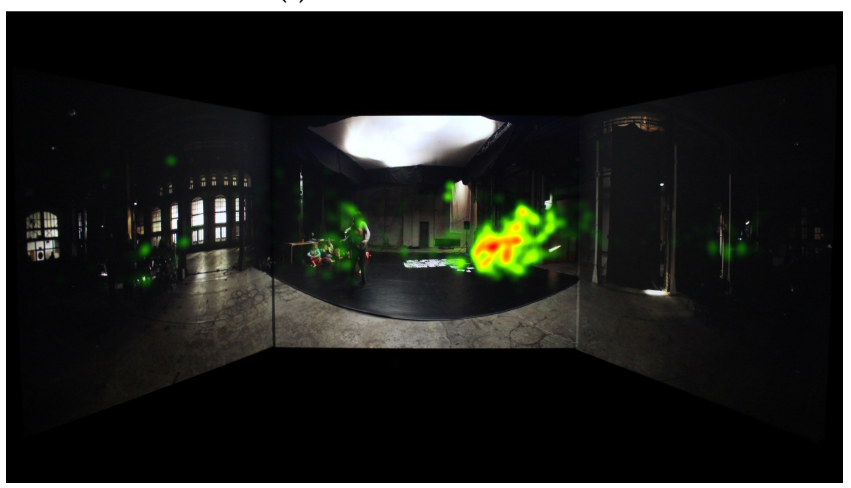

(d) 7.4.1 Sound Condition

Figure (4) Heat maps in the different soundtrack conditions 


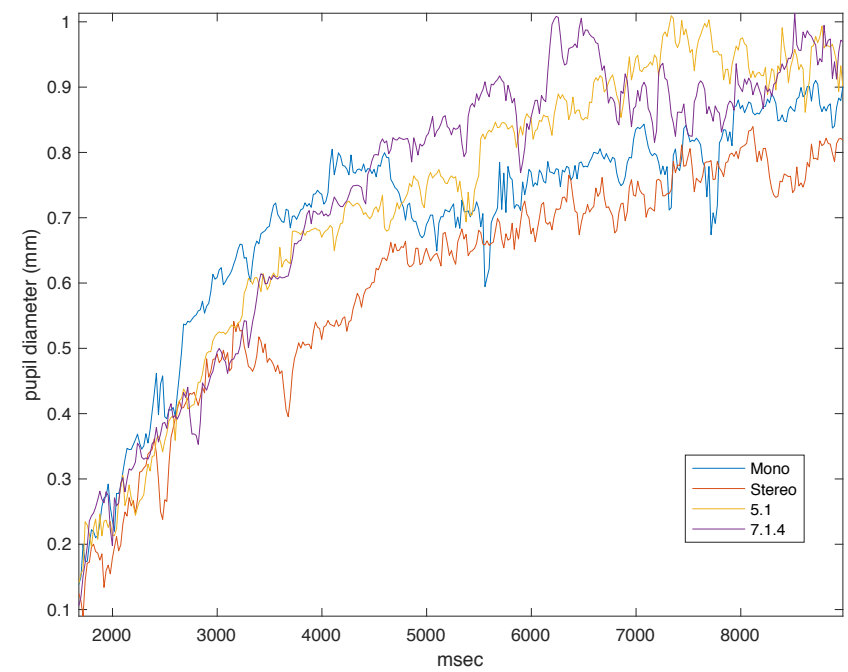

Figure (5) Average difference in pupil dilation, in $\mathrm{mm}$, since the beginning of the scene for each sound conditon.

controlling for time $\left(F_{(3,12)}=577.88, p=0.000\right)$. This test shows that the differences in progression throughout time per group are significant. Post-hoc pairwise comparison tests with a Scheffé correction show that the mono condition is different from all other conditions at the 0.001 level. The stereo condition is different form of mono and from 5.1 at 0.001 level and from 7.1.4 at 0.05 level. In 5.1 results are different from all other conditions at the 0.001 level. In 7.1.4 results are different from mono and 5.1 at 0.001 level and from the stereo at 0.05 . Based on these findings, it is possible to conclude that it was more difficult for the subjects to count the boxes in the 5.1 and 7.1.4 audio conditions. The stereo condition was the cognitively easiest sound condition.

\section{DISCUSSION}

The study reported here intended to analyze how different spatial sound configurations impact the attention of the user in an immersive audiovisual environment. We presented four groups of participants with a wide-screen projection of a video showing some human movement and some boxes falling from a pile onto the floor. All participants were asked to count how many boxes fell on the floor. Each group of participants experienced the video with a different soundtrack spatial configuration: mono(1 loudspeaker), stereo (two loudspeakers), 5.1 (six loudspeakers) and 7.4.1. (twelve loudspeakers).

Regarding the distribution of visual attention, a trend was found where the greater the spatial sound distribution, the larger the area in the screen that participants attended to. Subjects briefly explored (area with more that 100 fixations) $40.56 \mathrm{~m}^{2}$ more in the 7.4.1 condition, compared to the mono condition. The area with the greatest concentration of fixations was also larger in the more distributed sound conditions $\left(0.028 \mathrm{~m}^{2}\right.$ in the 5.1 and7.4.1 conditions), compared to the mono condition $\left(0.002 \mathrm{~m}^{2}\right)$. These results clearly show that participants move their eyes more and pay attention to a greater variety of visual elements when the sound is more distributed. This is not a novel finding, as it has been reported before
$[9,13]$, but it remains surprising. It would be expected that, when given a specific task, participants would be able to intentionally control their attention and focus on the relevant stimuli. What we observed here was that, while subjects did focus on the relevant screen areas, their pattern of visual behavior varied in the direction of greater distribution of fixations. This finding can point in the direction of greater difficulty in remaining focused on one area while sounds occur all around the user. Since this effect occurred in a selective attention task - where participants needed to focus on a narrow area of space- we assume that the effect found is automatic and involuntary. This is in line with some psychophysical and neurophysiological studies which have revealed that visual and auditory spatial information may be combined preattentively, leading to automatic shifts in attention caused by one sensory modality over the other $[4,5,7,8]$.

Regarding the pupil dilation metrics, this physiological measurement has rarely been applied to the study of user experience in multimedia reproductions. Pupil dilation is a measure of cognitive load: a theoretical construct describing the internal processing of tasks. The higher the cognitive load, the more mental resources must be allocated to a given task. For instance, a study found that adding subtitles to a movie decreased cognitive load [10]. Our study confirms that more distributed and immersive sound configurations lead to greater cognitive load when subjects are asked to pay attention to visual events. The more immersive sound conditions led to significantly higher pupil size, which might indicate that participants underwent greater cognitive effort to be able to complete the visual task. Interestingly, the stereo condition had significantly lower cognitive load than the remaining conditions, making it the best for this type of task.

The findings reported here should be taken into consideration when designing spatial sound for audiovisual environments. We do not dispute that more immersive sound will lead to greater presence or to greater perceived reproduction quality. However, frequently the audiovisual environments intend to also be able to pass on a message, which must be cognitively processed. When the goal is to actually understand and follow easily the contents displayed, then special attention should be paid to the sound design and to the audio setup. More surrounding sounds may lead to greater distraction or to more difficulty in selectively focusing on one central event. We advise the avoidance of spatially defined audio objects outside the area of visual interest as well as the reduction of the volume of peripheral sounds altogether during scenes where a main central event is present. More studies are needed to explore different sound configurations that could possibly retain some level of immersiveness without compromising the users' attention.

\section{ACKNOWLEDGMENTS}

We thank Synes Elischka for making available his 3D movie Egocure, part of which we adapted for the purpose of this study. This project received support from CPUP/FCT, project number UIDB/00050/2020. The research leading to these results has received funding from the European Union's Horizon 2020 research and innovation programme under the Marie Sklodowska-Curie grant no. 659114 and Aalto ARTS Supported Internship Programme. 


\section{REFERENCES}

[1] Javier Gómez Bolaños and Ville Pulkki. 2012. Immersive audiovisual environment with 3D audio playback. In Audio Engineering Society Convention 132. Audio Engineering Society.

[2] Dean Bullock. 2014. Dolby Atmos ${ }^{\circledR}$ Print Master File Specification. (2014).

[3] James J Cummings and Jeremy N Bailenson. 2016. How immersive is enough? A meta-analysis of the effect of immersive technology on user presence. Media Psychology 19, 2 (2016), 272-309.

[4] Jon Driver and Charles Spence. 1998. Crossmodal attention. Current opinion in neurobiology 8, 2 (1998), 245-253.

[5] Martin Eimer and Jose Van Velzen. 2002. Crossmodal links in spatial attention are mediated by supramodal control processes: evidence from event-related potentials. Psychophysiology 39, 4 (2002), 437-449.

[6] Synes Elischka. 2017. EgoCure-a narrative virtual reality short film. In Art and Virtual Reality.

[7] Jessica J Green and John J McDonald. 2006. An event-related potential study of supramodal attentional control and crossmodal attention effects. Psychophysiology 43, 2 (2006), 161-171.

[8] Jessica J Green, Wolfgang A Teder-Sälejärvi, and John J McDonald. 2005. Control mechanisms mediating shifts of attention in auditory and visual space: a spatiotemporal ERP analysis. Experimental Brain Research 166, 3-4 (2005), 358-369.
[9] Victoria Korshunova, Gerard B Remijn, Synes Elischka, and Catarina Mendonca. 2019. The Impact of Sound Systems on the Perception of Cinematic Content in Immersive Audiovisual Productions. In 2019 12th Asia Pacific Workshop on Mixed and Augmented Reality (APMAR). IEEE, 1-5.

[10] Jan-Louis Kruger, Esté Hefer, and Gordon Matthew. 2013. Measuring the impact of subtitles on cognitive load: Eye tracking and dynamic audiovisual texts. In Proceedings of the 2013 Conference on Eye Tracking South Africa. 62-66.

[11] John J McDonald, Wolfgang A Teder-SaÈlejaÈrvi, and Steven A Hillyard. 2000. Involuntary orienting to sound improves visual perception. Nature 407, 6806 (2000), 906-908.

[12] Catarina Mendona and Victoria Korshunova. 2020. Surround Sound Spreads Visual Attention and Increases Cognitive Effort in Immersive Media Reproductions. https://doi.org/10.5281/zenodo.3945057

[13] Catarina Mendonça, Olli Rummukainen, and Ville Pulkki. 2015. 3D sound can have a negative impact on the perception of visual content in audiovisual reproductions. Georgia Institute of Technology.

[14] Olli Rummukainen and Catarina Mendonça. 2016. Task-relevant spatialized auditory cues enhance attention orientation and peripheral target detection in natural scenes. (2016).

[15] Pauline van der Wel and Henk van Steenbergen. 2018. Pupil dilation as an index of effort in cognitive control tasks: A review. Psychonomic bulletin \& review 25, 6 (2018), 2005-2015. 